\title{
Publisher's Note: Laser stabilization using saturated absorption in a cavity-QED system [Phys. Rev. A 92, 013817 (2015)]
}

D. A. Tieri, J. Cooper, Bjarke T. R. Christensen, J. W. Thomsen, and M. J. Holland (Received 11 September 2015; published 17 September 2015)

DOI: 10.1103/PhysRevA.92.039902

PACS number(s): 42.62.Fi, 32.80.Wr, 37.30.+i, 42.50.Ct, 99.10.Fg

This paper was published online on 10 July 2015 with errors throughout the article. In Eq. (3) the third line should read as

$$
+\frac{1}{2 T_{2}} \sum_{j=1}^{N}\left\{\hat{\sigma}_{j}^{z} \hat{\rho} \hat{\sigma}_{j}^{z}-\hat{\rho}\right\},
$$

The second line of text after Eq. (3) should read as "emission rate for the atoms, and $\frac{1}{T_{2}}$ is the inhomogeneous dephasing rate." The text following Eq. (6) should read as "where $\gamma_{p} \equiv \frac{1}{T_{2}}+\frac{\gamma}{2}$ is the total decay rate of the atomic dipole." The paper has been corrected as of 21 August 2015. The text and equations are incorrect in the printed version of the journal. 